\title{
Enhanced Secretion of Endothelin by Endothelial Cells in Response to Hemoglobin
}

\author{
Isao Fuwa, Marc MAYBERG, Corinne Gadjusek, Tsutomu HARAdA \\ and Zhengyu LUO
}

Department of Neurosurgery, University of Washington, Seattle, Washington, U.S.A.

\begin{abstract}
Confluent cultures of bovine aortic endothelium in serum-free medium were exposed to increasing concentrations $\left(10^{-6}-10^{-4} \mathrm{M}\right)$ of freshly prepared erythrocyte lysates (primarily hemoglobin). Hemoglobin increased endothelin-1 secretion into the medium in a dose-dependent manner after 24 hours. The enhanced secretion of endothelin- 1 in response to hemoglobin was sustained for 72 hours, suggesting active production and secretion of endothelin-1 rather than release from intracellular pools. Secreted endothelin-1 in the medium was characterized by high-performance liquid chromatography coupled with radioimmunoassay. Endothelin-1, a potent and long-lasting vasoconstrictor, may be one of the causative factors of cerebral vasospasm after subarachnoid hemorrhage. Oxyhemoglobin, derived from periarterial clot, may play an important role in the secretion of endothelin-1 in cerebral vasospasm.
\end{abstract}

Key words: vasospasm, subarachnoid hemorrhage, endothelin, hemoglobin, endothelial cell

\section{Introduction}

Cerebral vasospasm is a poorly understood phenomenon characterized by sustained narrowing of cerebral arteries occurring 5-10 days after subarachnoid hemorrhage. ${ }^{13)}$ Chronic arterial narrowing after subarachnoid hemorrhage is associated with a variety of structural and physiological changes in the arterial wall involving both endothelial and smooth muscle cells. ${ }^{13,191}$ Numerous changes have been demonstrated in cerebral endothelium after exposure to subarachnoid blood, including alterations in prostaglandin metabolism, increased permeability and diminished secretion of endothelium-dependent relaxation factor (EDRF). ${ }^{11,19)}$ Although severe vasoconstriction per se may affect endothelial cell physiology and structure, a variety of substrates released from periarterial clot may also directly mediate endothelial function. ${ }^{11,13)}$ Although the precise mechanism causing cerebral vasospasm has not been determined, considerable evidence suggests that constituents of erythrocyte cytosol, especially oxyhemoglobin, are critical in the develop- ment of delayed arterial narrowing. ${ }^{11,13)}$

Endothelin-1 (ET-1) is a long-lasting, potent vasoconstrictor secreted by vascular endothelial cells. ${ }^{21)}$ Both plasma and cerebrospinal fluid ET concentrations were increased in patients after subarachnoid hemorrhage, ${ }^{12)}$ and intracisternal injection of ET produced prolonged narrowing of cerebral arteries in vivo. ${ }^{2,9,14)}$ Nevertheless, a direct relationship between hemoglobin exposure and ET secretion has not been identified. In this experiment, bovine aortic endothelial cells in vitro were treated with fresh erythrocyte lysate and secretion of ET into the medium determined by radioimmunoassay (RIA).

\section{Materials and Methods}

\section{Cell culture}

Bovine aortic endothelial cells were isolated and grown as previously described. ${ }^{6}$ Cells were subcultured in Waymouth's medium (Gibco, Grand Island, N.Y., U.S.A.) supplemented with sodium bicarbonate, nonessential amino acids, sodium pyruvate and $10 \%$ serum. Cells were passaged in $75 \mathrm{~cm}^{2}$

Received November 2, 1992; Accepted March 23, 1993

Author's present address: I. Fuwa, M.D., Division of Neurosurgery, Minamata General Medical Center, Minamata, Kumamoto, Japan. 
flasks with $0.05 \%$ trypsin at a split ratio of $1: 3$ and used in experiments from passages 10-20.

\section{Preparation of erythrocyte lysate}

Fresh human blood was heparinized $(20 \mathrm{U} / \mathrm{ml})$ and centrifuged under sterile conditions at $2000 \mathrm{G}$ for 5 minutes. After removal of buffy coat and platelet-rich plasma, the remaining erythrocytes were resuspended in phosphate buffer solution (PBS) (pH 7.4), centrifuged as above three times, and PBS plus residual buffy coat removed by pipetting. Washed erythrocytes were lysed with an ultrasonic disrupter, centrifuged at $100,000 \mathrm{G}$ for 2 hours, and the cytosol fraction (hemoglobin) was separated from the erythrocyte membrane pellet by pipetting. The concentrations of total and oxyhemoglobin were measured with a co-oxymeter, and adjusted fresh lysate solutions $10^{-3}-10^{-5} \mathrm{M}$ were stored at $-70^{\circ} \mathrm{C}$ until application.

\section{Preparation of conditioned medium}

Endothelial cells were planted at a density of 70,000 cells per well in 48-well plates (Falcon Labware, Oxnard, Cal., U.S.A.) and allowed to grow to confluency (3 days). At confluency, the medium was changed to serum-free Waymouth's medium. $25 \mu \mathrm{l}$ aliquots of hemoglobin solution (above) were added at concentrations of $10^{-6}-10^{-4} \mathrm{M}$, with similar volumes of medium as control. After incubation from 24 to 72 hours, conditioned media were obtained and stored at $-70^{\circ} \mathrm{C}$ for RIA measurement. Control solutions of thrombin (Sigma, St. Louis, Mo., U.S.A.) $5 \mathrm{U} / \mathrm{ml}$ were applied for 24 hours using the same procedure.

\section{Preparation of ${ }^{125}$ I-labeled ET-1 and RIA}

Iodination of ET-1 used the lactoperoxidase method, ${ }^{8)}$ mixing ${ }^{125} \mathrm{I}-\mathrm{Na}$ (New England Nuclear, Boston, Mass., U.S.A.) and porcine/human ET-1 (Peninsula Laboratories, Belmont, Cal., U.S.A.). RIA was carried out in buffer containing $0.1 \mathrm{M}$ sodium phosphate buffer, $0.05 \mathrm{M} \mathrm{NaCl}, 0.1 \%$ bovine serum, $0.01 \%$ sodium azide and $0.1 \%$ Triton $\mathrm{X}-100$ at $\mathrm{pH}$ 7.4. Conditioned medium $(100 \mu \mathrm{l})$ or standards (human/porcine ET-1, Peninsula Laboratories; 10$400 \mathrm{pg} /$ tube) were mixed with $50 \mu \mathrm{l}$ of rabbit antiET-1 serum (Peninsula Laboratories; 1:1000) in buffer solution. Mixtures were incubated for 24 hours at $4^{\circ} \mathrm{C}$ and $50 \mu \mathrm{l}$ of ${ }^{125}$ l-labeled ET-1 $(10,000$ cpm) was added. After a second 24-hour incubation, $10 \mu \mathrm{l}$ of normal rabbit serum (Calbiochemical, San Diego, Cal., U.S.A.), $20 \mu$ l of goat anti-rabbit immunoglobulin $\mathrm{G}$ serum (Calbiochemical) and $10 \%$ polyethylene glycol (Sigma) were added and vortex- ed. The precipitate was collected by centrifugation at $3000 \mathrm{rpm}$ for 30 minutes and radioactivity was determined by a gamma counter.

\section{High-performance liquid chromatography (HPLC)}

Confluent endothelial cells were incubated in serum-free conditioned medium for 24 hours, and collected in $1 \mathrm{~N}$ acetic acid. HPLC (Pharmacia LKB Biotechnology, Piscataway, N.J., U.S.A.) was performed with a single solvent system (flow rate $0.5 \mathrm{ml} / \mathrm{min}$ ) and $1 \mathrm{ml}$ fractions were collected. The elutes were lyophylized, dissolved in the assay buffer and measured with the RIA as above. Control standards of ${ }^{125}$ I-labeled ET-1 in endothelial cell medium were eluted by the same procedure and fractions counted with a gamma counter.

\section{Statistical analysis}

Media from five wells for each experimental group were independently assayed for ET-1 content. Data from each group were pooled and expressed as mean \pm SD. ET-1 levels for experimental groups were compared to control levels at each time period using the $t$-test, and a $\mathrm{p}$ value less than 0.05 considered statistically significant.

\section{Results}

The co-oxymeter measurements showed that the fresh erythrocyte lysate solution contained $95 \%$ of

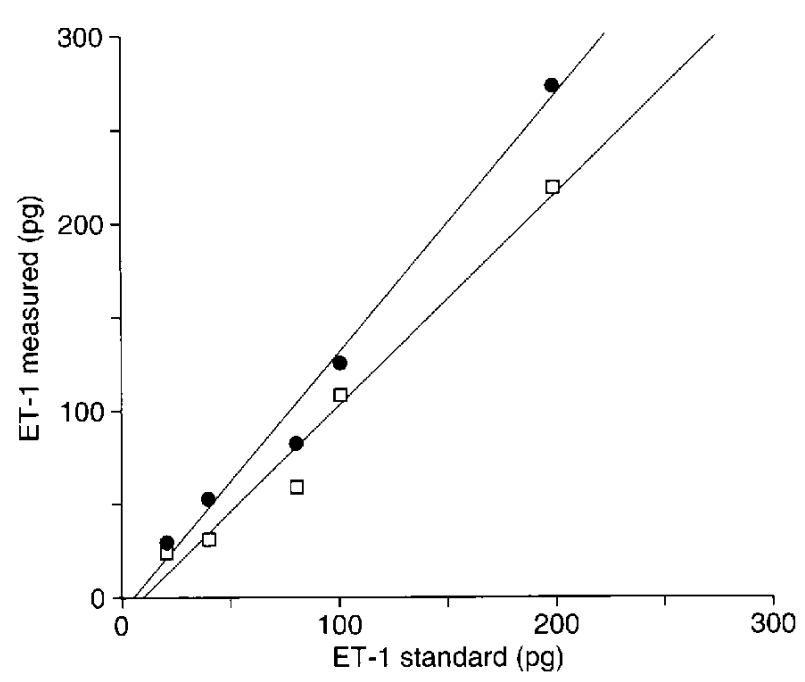

Fig. 1 RIA of ET-1 in media with $(\bullet)$ or without $(\square)$ fresh erythrocyte lysate (hemoglobin). Hemoglobin $\left(10^{-4} \mathrm{M}\right)$ did not significantly affect the immunoreactive ET-1 measurement. 


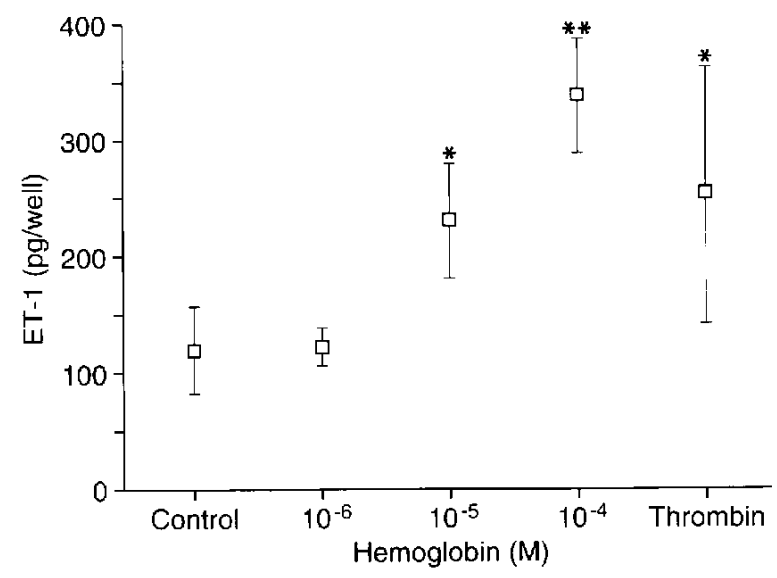

Fig. 2 Effect of hemoglobin on ET-1 secretion into cultured medium over 24 hours. Hemoglobin increased ET-1 secretion in a dose-dependent manner between $10^{-6}$ and $10^{-4} \mathrm{M}$ at levels equivalent to those observed for thrombin ( 5 $\mathrm{U} / \mathrm{ml}$ ). Data are expressed as mean $\pm \mathrm{SD}$. ${ }^{*} \mathrm{p}<0.05,{ }^{* *} \mathrm{p}<0.01$.

oxyhemoglobin. No detectable immunoreactive ET1 was present in the hemoglobin solution. Addition of hemoglobin $\left(10^{-4} \mathrm{M}\right)$ to standard solutions of ET1 did not significantly affect the RIA measurements (Fig. 1).

Endothelial cells treated for 24 hours with hemoglobin showed a dose-dependent increase in ET-1 secretion. In comparison to the control, $10^{-4} \mathrm{M}$

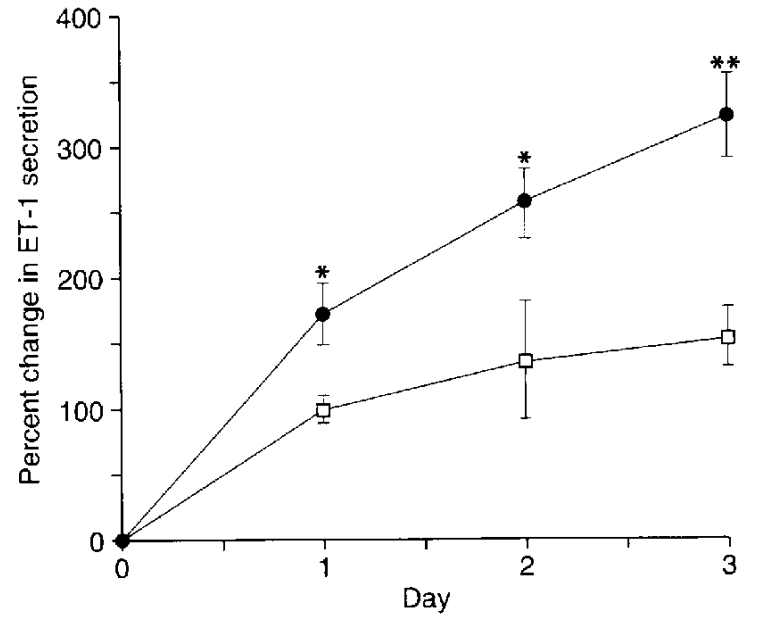

Fig. 3 Time course of ET-1 secretion in media with (Q) or without ( $\square$ ) hemoglobin $\left(10^{-5} \mathrm{M}\right)$. Enhanced secretion of ET-1 in response to hemoglobin was sustained for 72 hours. Data are expressed as the mean $\pm \mathrm{SD}$ percent change in ET-1 levels compared to the ET-1 level without hemoglobin at 24 hours. ${ }^{*} \mathrm{p}<$ $0.05,{ }^{* *} \mathrm{p}<0.01$.

of hemoglobin produced a two-fold increase in ET-1 in the medium $(p<0.01)$, with levels comparable to thrombin $(5 \mathrm{U} / \mathrm{ml})$ stimulation (Fig. 2). The enhanced secretion of ET-1 in response to hemoglobin $\left(10^{-5} \mathrm{M}\right)$ was sustained for 72 hours, with two-fold

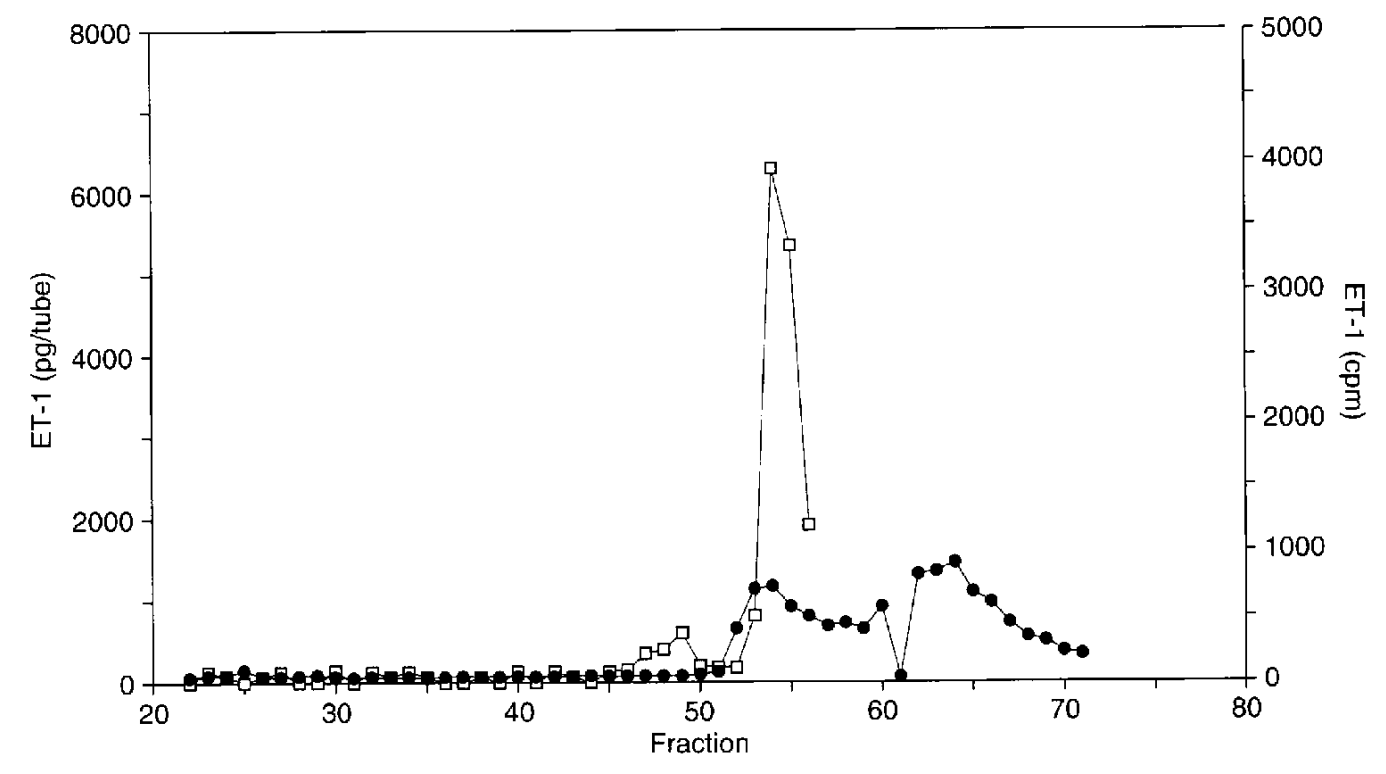

Fig. 4 HPLC/RIA for ET-1. Immunoreactive ET-1 in cultured medium ( $\square$ ) eluted as a single component corresponding to synthetic ${ }^{125}$ I-labeled ET-1 radioactivity $(\bullet)$. 
increase in levels at that time compared to controls (Fig. 3). By HPLC, ET-1 in endothelial cell medium eluted as a single peak coincident with synthetic radioactive ET-1 (Fig. 4).

\section{Discussion}

Yanagisawa et $a l^{211}$ first isolated and identified a potent, long-acting vasoconstricting agents from vascular endothelial cells which was termed endothelin. The ET family includes ET-1, ET-2 and ET-3. The vasoconstrictor activity of ET-1, a peptide containing 21 amino acids, appears to be more potent and more long lasting than any other known vasoconstrictor. Preproendothelin, which contains 203 amino acid residues, is cleaved by an endopeptidase to produce big ET, containing 39 residues. ET-1 is derived from ET by a putative "ET-converting enzyme." 1,21 )

In our study, the lysate of human erythrocytes (primarily oxyhemoglobin) markedly enhanced the basal secretion of ET-1 in cultured bovine aortic endothelial cells. The accuracy of RIA for ET-1 was unaffected by the presence of a high concentration of hemoglobin. HPLC coupled with RIA revealed that the major component of immunoradioactive ET-1 in the endothelial cell medium corresponded to synthetic ET-1. Because the antibody used in this study cross reacts with ET-1 and big ET-1, some components of the immunoreactive substrate secreted by endothelial cells may be the latter peptide, which is less vasoactive compared to ET-1. ${ }^{10}$

Endothelial secretion or synthesis of ET-1 in vitro has been stimulated by a variety of agents, including thrombin, ${ }^{21)}$ epinephrine, ${ }^{21)}$ transforming growth factor $\beta,{ }^{17)}$ vasopressin, ${ }^{4)}$ endotoxin ${ }^{20)}$ and hypoxia. ${ }^{18)}$ ET-1 is secreted by a constitutive pathway directly from the Golgi apparatus and/or microsomes. ${ }^{16)}$ Both phorbor ester and calcium ionophore stimulated endothelial ET secretion, suggesting that a calcium-mediated mechanism is involved in this process. ${ }^{4}$ ET-1 is not stored in cytoplasmic granules and its production is regulated predominantly at the level of messenger ribonucleic acid transcription. ${ }^{21}$ In our experiment, the enhanced secretion of ET-1 in response to hemoglobin was sustained for 72 hours, suggesting active synthesis and secretion of ET-1 rather than release from intracellular pools.

Hemoglobin may stimulate endothelial ET-1 secretion by a number of mechanisms. (Oxy)hemoglobin catalyzes the formation of the oxygen-free radical (superoxide), which initiates a cascade of cytotoxic lipid peroxidation from unsaturated fatty acids in the cell membrane..$^{5,71)}$ In addition, hemoglobin stimulates prostaglandin synthesis and release from endothelial cells. ${ }^{5}$ Superoxide dismutase, a scavenger of superoxide anions, inhibits thrombin-induced endothelial ET-1 secretion. ${ }^{3)}$ Alternatively, hemoglobin may influence ET-1 secretion by its interaction with EDRF. Several authors have postulated an inverse relationship in levels of ET-1 and EDRF secreted by endothelium, ${ }^{3,4)}$ although Ohlstein et al. ${ }^{17)}$ showed that release of EDRF increased in response to ET-1. Hemoglobin is a potent inhibitor of extracellular EDRF. ${ }^{11)}$ By this means, extracellular hemoglobin may inhibit autocrine EDRF influence upon endothelial cells, thereby augmenting ET-1 secretion. ${ }^{3)}$

Increased plasma and cerebrospinal fluid concentrations of ET-1 were reported in humans after subarachnoid hemorrhage, although elevated circulating ET-1 levels have been observed in other acute illnesses such as myocardial infarction. ${ }^{15}$ ) Cisternal injection of ET-1 induced prolonged basilar artery narrowing in animal models with subsequent histological changes in arterial walls similar to those observed after subarachnoid hemorrhage. ${ }^{9,14)}$ Although intracisternal injection produced arterial narrowing at extremely low doses of ET-1 (10 pM), ${ }^{2)}$ intraarterial injection did not cause vasoconstriction in cats. ${ }^{15 !}$ Although normal cerebral arteries are impermeable to ET-1, alterations in the blood-arterial barrier after subarachnoid hemorrhage may allow ET-1 from luminal endothelium to penetrate into the vascular wall. ${ }^{9,19)}$ Conversely, the adventitia and media of cerebral arteries are freely permeable to large molecules in the subarachnoid space, ${ }^{22)}$ thus enabling hemoglobin from lysed perivascular erythrocytes to access endothelial cells at concentrations analogous to those observed in vivo.

In summary, cultured bovine vascular endothelial cells secreted ET-1 into the medium in a dose- and time-dependent manner after exposure to fresh erythrocyte lysate. Augmentation of ET-1 release by hemoglobin over 72 hours suggested stimulation of ET-1 synthesis, rather than secretion from intracellular pools. Hemoglobin may play an important role in the secretion of ET-1 in cerebral vasospasm.

\section{Acknowledgments}

The authors would like to acknowledge the secretarial assistance of Ms. Mimi Platt.

This work was supported in part by National Institute of Health Grant \#HL44458. 


\section{References}

1) Änggard E, Botting R, Vane J: Endothelins. Blood Vessels 27: 269-281, 1990

2) Asano T, Ikegaki I, Suzuki $Y$, Satoh S, Shibuya M: Endothelin and the production of cerebral vasospasm in dogs. Biochem Biophys Res Commun 159: 1345-1351, 1989

3) Boulanger $C$, Luscher $T$ : Release of endothelin from the porcine aorta. $J$ Clin Invesi 85: 587-590, 1990

4) Emori $T$, Hirata $Y$, Ohta $K$, Schichiri M, Marumo F: Secretary mechanism of immunoreactive endothelin in cultured bovine endothelial cells, Biochem Biophys Res Commun 160: 93-100, 1989

5) Feola M, Simoni J, Fishman D, Tran R, Canizaro P: Biocompatibility of hemoglobin solutions. 1. Reactions of vascular endothelial cells to pure and impure hemoglobins. Artif Organs 13: 209-215, 1989

6) Gajdusek C, Schwartz S: Technique for cloning bovine aortic endothelial cells. In Vitro 19: 394-402, 1983

7) Harada $T$, Mayberg M: Inhibition of delayed arterial narrowing by the iron-chelating agent deferoxamine. $J$ Neurosurg 77: 763-767, 1992

8) Hirata Y, Yoshimi H, Takata S: Cellular mechanism of action by a novel vasoconstrictor endothelin in cultured rat vascular smooth muscle cells. Biochem Biophys Res Commun 154: 868-875, 1988

9) Kobayashi H, Hayashi M, Kobayashi S: Cerebral vasospasm and vasoconstriction caused by endothelin. Neurosurgery 28: 673-679, 1991

10) Kohno M, Yasunari K, Murakawa K: Release of immunoreactive endothelin from porcine aortic strips. Hypertension 15: 718-723, 1990

11) Macdonald R, Weir B: A review of hemoglobin and the pathogenesis of cerebral vasospasm. Stroke 22: 971-982, 1991

12) Masaoka H, Suzuki R, Hirata $Y$, Emori T, Marumo $\mathrm{F}$, Hirakawa K: Raised plasma endothelin in aneurysmal subarachnoid hemorrhage. Lancet 2: 1402,1989

13) Mayberg M, Okada T, Bark D: Morphological changes in cerebral arteries after subarachnoid hemor- rhage. Neurosurg Clin N Am 1: 417-432, 1990

14) Mima $T$, Yanagisawa $M$, Shigeno $T$ : Endothelin acts in feline and canine cerebral arteries from the adventitial side. Stroke 20: 1553-1556, 1989

15) Miyauchi $T$, Yanagisawa $M$, Tomizawa $T$ : Increased plasma concentrations of endothelin-1 and big endothelin-1 in acute myocardial infarction. Lancet 2: $53-54,1989$

16) Naruse $M$, Naruse $K$, Kurino F: Radioimmunoassay for endothelin and immunoreactive endothelin in culture medium of bovine endothelial cells. Biochem Biophys Res Commun 160: 662-668, 1989

17) Ohlstein E, Storer B, Butcher J, Debouck C, Feuerstein G: Platelets stimulate expression of endothelin mRNA and endothelin biosynthesis in cultured endothelial cells. Circ Res 69: 832-841, 1991

18) Rakugi $H$, Tabuchi $Y$, Nakamura $M$ : Evidence for endothelin-1 release from resistance vessels of rats in response to hypoxia. Biochem Biophys Res Commun 169: 973-977, 1990

19) Sasaki T, Kassel NF, Yamashita M, Fujiwara S, Zuccarello $\mathrm{M}$ : Barrier disruption in the major cerebral arteries following experimental subarachnoid hemorrhage. $J$ Neurosurg 63: 433-440, 1985

20) Sugiura $M$, Inagami $T$, Kon $V$ : Endotoxin stimulates endothelin-release in vivo and in vitro as determined by radioimmunoassay. Biochem Biophys Res Commun 161: 1220-1227, 1989

21) Yanagisawa M, Kurihara H, Kimura S: A novel potent vasoconstrictor peptide produced by vascular endothelial cells. Nature 332: 411-415, 1988

22) Zervas N, Liszczak $T$, Mayberg M, Black P: Cerebrospinal fluid may nourish cerebral vessels through pathway in the adventitia that may be analogues to systemic vasa vasorum. J Neurosurg 56 : $475-481,1982$

Address reprint requests to: I. Fuwa, M.D., Division of Neurosurgery, Minamata General Medical Center, 12-1 Tenjin-cho, Minamata, Kumamoto 867, Japan. 